\title{
Caviar con Ron: 'Sdelano na kube'
}

\author{
Caviar with Rum: 'Sdelano na kube’
}

\section{JACQUELINE LOSS}

University of Connecticut · jacqueline.loss@uconn.edu

Profesora de Literatura Latinoamericana, Literatura Comparada y Estudios Culturales. Ha publicado Dreaming in Russian: The Cuban Soviet Imaginary y Cosmopolitanisms and Latin America: Against the Destiny of Place. Es coeditora, junto a José Manuel Prieto, de Caviar with Rum: Cuba-USSR and the Post-Soviet Experience y con Esther Whitfield de New Short Fiction from Cuba.

\section{José Manuel Prieto}

Seton Hall University•jose.prieto@shu.edu

Además de profesor e investigador es autor de varias novelas y traductor de literatura rusa al español. Entre sus libros destacan Rex, Livadia, Enciclopedia de una vida en Rusia, el conjunto de relatos El Tartamudo y la rusa o el libro de viajes Treinta días en Moscú.

RECIBIDO: 26 DE ENERO DE 2015

ACEPTADO: 4 DE JUNIO DE 2015

Resumen: Los autores analizan la gama vasta de actitudes hacia lo soviético -desde una minimización del impacto soviético en Cuba a una expresión exagerada de gratitud hacia los "rusos"- que se han encontrado desde que comenzaron su colaboración investigativa en 2004 y que tuvo un punto álgido con la publicación de Caviar with Rum: Cuba-USSR and the Post-Soviet Experience (2012). En este texto, buscan compartir su experiencia, relatar sus principales etapas y el estado actual de la cuestión del imaginario cubano de lo soviético y lo ruso.

Palabras clave: Cuba, URSS, Post-soviético.
Abstract: The authors analyze the vast range of attitudes toward the Soviet -from the minimization of the Soviet impact in Cuba to an exaggerated expression of gratitude toward the "Russians" - that they have encountered since they began their investigative collaboration in 2004, that had a high point on the publishing of Caviar with Rum: CubaUSSR and the Post-Soviet Experience (2012). In this text, they seek to share their experience, telling the first stages and the current state of the question of the Cuban imaginary of the Soviet and the Russian.

Keywords: Cuba, USSR, Post-Soviet.

DOI: 10.7203/KAM.5.4641 
Jacqueline Loss y José Manuel Prieto. Caviar con ron...

\section{Historia y nostalgia}

En el 2000, cuando Vladimir Putin viajó a Cuba, la primera visita de un presidente ruso desde Mijaíl Gorbachov en 1989, la influencia rusa en la isla parecía apenas un recuerdo. "No quedó nada, no bailamos como los rusos, no comemos como los rusos, y ni siquiera bebemos vodka”, le dijo una cubana a los periodistas. Todo parece indicar que Vladimir Putin no reparó en tales detractores cuando en julio del 2014 decidió comenzar su nueva gira histórica por América Latina con su visita a Cuba poco después de haberle condonado el $90 \%$ de los 35.2 mil millones dólares de la antigua deuda cubana al extinto gobierno soviético. Un acto que días después la presidenta de Argentina, Cristina Fernández de Kirchner, calificó de ejemplo a seguir para otros países. A pesar de éste y otros gestos, encaminados a mejorar las relaciones en muchos campos, incluido el militar, la mayoría de los expertos coincidieron en que los planes del gobierno ruso para relanzar la ex base soviética de espionaje en la localidad de Lourdes, en las afueras de la Habana, no presentaba una amenaza real para los Estados Unidos. Para muchos, aquellos gestos, que recordaban la antigua grandilocuencia soviética, no eran sino una suerte de pantalla que más que nada evidenciaba una "nostalgia imperialista" por parte de Putin, estrategias para el público con las que buscaba paliar los problemas reales que enfrentaba y todavía enfrenta en su propio país.

Se pueden identificar nostalgias semejantes a la de Putin en varios momentos de la historia de la actual amistad cubano-rusa, algo que se vincula a lo que Svetlana Boym, la célebre teórica de lo postsoviético, ha llamado "nostalgia restaurativa", término que se refiere a la búsqueda de la "reconstrucción transhistórica del hogar perdido" (xviii). Al comienzo del matrimonio cubano-soviético, en los lejanos años sesenta, varios personajes encarnaron este sentimiento. En 1967, el conocido escritor cubano Samuel Feijóo publicó una relación de su viaje a la URSS tres años antes en la que afirmó: "Hasta hoy no hay nostalgia de mi país, amigos, etc. Sigo en mi tierra" (108). No menos sorprendente fue el comentario de la archiconocida primera mujer cosmonauta, Valentina Tereshkova, quien de visita en Cuba, en 1963, fue llevada a una textilera de la isla donde, en plena euforia, entonó la muy conocida por todos los rusos melodía "Kuba, liubov moia", "Cuba, mi amor". "Me sentí en Cuba como en mi propia casa”, añadió. Aunque es difícil imaginar que un cubano expresara una emoción similar a la de Feijóo hoy día, o bien que cualquiera de los miles de turistas rusos que visitan hoy la isla expresasen un sentimiento igual al de la célebre heroína del espacio, es innegable que aunque disminuida, la "pompa" de la fraternal amistad soviética-cubana vive ahora un segundo tiempo. ${ }^{1}$ Lo que es más, sigue sirviendo a la geopolítica del siglo XXI, bien sea para consolidar la verdad sobre el rol que el desaparecido país jugó en la supervivencia de la isla ante el embargo estadounidense, bien para revestirla con el nuevo ropaje más propio del siglo XXI

\footnotetext{
${ }^{1}$ Véase "Russian Nostalgia Nights in Cuba Threatened by U.S. Détente".
} 
bajo, por ejemplo, la apariencia de novísimos experimentos gastronómicos como el restaurante Nazdarovie de La Habana, que "[celebra] el lazo social y cultural que nació entre el pueblo cubano y los pueblos de la ex Unión Soviética... [nutriéndose] de las memorias y recuerdos de los miles de cubanos que pasaron muchos años de su juventud estudiando en la URSS”.

De modo que puede decirse, y es algo que varios artículos recientes sobre el turismo en Cuba lo atestiguan, que el negocio del pasado soviético está muy vivo. Sea a través de la gastronomía, como ya hemos dicho, sea alquilando una limosina soviética para dar un giro de autenticidad a ciertos aspectos de la vida nocturna habanera, sea escuchando viejos himnos soviéticos "transidos de nostalgia", el consumo de este lazo histórico es muy diferente hoy en día, que hace una década cuando Jacqueline Loss y José Manuel Prieto anunciaron su colaboración en el estudio de la complicada memoria de Cuba/URSS. Un esfuerzo que al comienzo encontró cierta resistencia caracterizada en la frase "aquí los rusos no dejaron nada" que tiene resonancias hasta en El humor de Misha: la crisis del 'socialismo real' en el chiste político, el ensayo que se puede considerar representativo de un momento importante en la memorialización cubana de "rusos" publicado por el ex ministro de cultura Abel Prieto en 1997:

En Cuba nadie hubiera podido concebirse como habitante de un satélite de la URSS: había y hay entre nosotros una percepción demasiado vívida, y permanentemente renovada, de la absoluta independencia de la dirección revolucionaria, como para convivir con una idea semejante...

Aunque hubo zonas de la cultura y la educación que sufrieron la dañina influencia del copismo, en el arte y la literatura esta resonancia fue muchísimo menor (99).

Hemos tropezado con una gama vasta de actitudes hacia lo soviético: desde la minimización del impacto soviético en Cuba a una expresión exagerada de gratitud hacia los rusos. Estas transformaciones en la memorialización, de una negación rotunda a una aceptación actual que no dudaría de llamar entusiasta fue algo que no pudimos anticipar del todo cuando decidimos organizar el que puede considerarse el primer simposio internacional dedicado a estudiar el impacto del vínculo soviético en Cuba, y la publicación del libro Caviar with Rum: Cuba-USSR and the post-Soviet Experience, que aquilató la huella de esa, extraña relación de un país del tercer mundo con una potencia lejana y virtualmente desconocida, todo dentro del marco de la Guerra Fría. En el texto que el lector tiene en sus manos, buscaremos compartir esta experiencia, relatar sus principales etapas y el estado actual de la cuestión. 
Jacqueline Loss y José Manuel Prieto. Caviar con ron...

\section{El simposio}

Todo comenzó una noche de comienzos de 2004, cuando José Manuel Prieto, novelista cubano y Jacqueline Loss, académica estadounidense, ensayista y especialista en temas cubanos, coincidimos en una reunión de amigos en la ciudad de Nueva York. En ocasiones anteriores habíamos abordado el tema de la presencia soviética en Cuba porque como es sabido, uno de nosotros, Prieto, había pasado una decena de años en aquel país y escrito novelas ambientadas en Rusia, mientras que desde hacia un tiempo el tema de la presencia soviética en la isla había sido del interés profesional de Loss. Pero fue justo esa noche de un intenso frío casi siberiano, que convenimos en hacer un simposio que abordara aquel tema desde los más disimiles ángulos y con la más amplia perspectiva, de modo que desmintiera aquel aserto, que considerábamos -y cuánta razón teníamos, como se verá- equivocado. Para esto el simposio debería, además, reunir a académicos y pensadores de varios países, así como a creadores cubanos y no cubanos, unidos todos por el objetivo de abordar de la manera más seria y enjundiosa la memoria de las largas y complicadas relaciones entre Cuba y la URSS.

Como ya hemos dicho, a la fecha, invierno del 2004, y casi quince años luego del enfriamiento de las relaciones entre Cuba y la URSS, no se había dado ningún diálogo intelectual que abordara el tema con una amplitud adecuada o el rigor necesario. De todos, sin embargo, era conocida la crisis en que se sumió el país cuando la URSS cercenó el cordón umbilical de su generosa ayuda a la isla, la catastrófica situación en que se sumió el país. Entonces, por pura deducción matemática, algo había estado ocurriendo de suma importancia, tanto económica como cultural, algo que valía la pena indagar, de qué modo y a qué profundidad se había dado esa alianza y sobre todo, cómo ahora, más de un decenio después, se recordaban aquellos años tan importantes para la isla en que la Unión Soviética había fungido de "hermano mayor", tanto en lo ideológico como en lo económico y en tantos y tantos otros ámbitos. Los dos coincidimos que ya era hora de organizar tal simposio, encauzar un diálogo. No fuimos capaces de imaginar, sin embargo, cuán ardua sería la tarea que nos habíamos impuesto.

El principal obstáculo que encontramos al comienzo mismo fue lograr convencer a nuestros posibles patrocinadores cubanos en los Estados Unidos de la existencia y legitimidad del tema, que valía la pena dedicarle no ya una mera reflexión académica en forma de ensayo o bien en una entrevista a fondo, sino el simposio que teníamos en mente, que un esfuerzo multidisciplinario que suscitara un análisis a fondo era lo más adecuado. Lo cierto es que era entendible. O bien, lo cierto es que era entendible hasta cierto punto. Muchas de las personas que contactamos, así como un gran número de cubanos dentro y fuera de la isla con quienes hablamos de nuestro proyecto, -y esta es una percepción que nuestro simposio ayudó a explorar- sentían que su país había sido colonizado por la Unión Soviética, una potencia extranjera, un país, por lo demás, con el cual ni sus padres ni ellos mismos habían tenido 
afinidad alguna. Nuestros posibles patrocinadores cubanos en los Estados Unidos no podían creer que deseáramos revisitar intelectualmente este periodo en la historia cubana. Como si por el acto de estudiarlo, los soviéticos -que para ellos sufrían una falta de estética aparte de que no compartían sus valores capitalistas- regresarían al escenario. Por otra parte, nadie parecía querer admitir, algo que notamos a los más disímiles niveles, que aquella larga interacción había tenido profundas consecuencias culturales y sociopolíticas. Lógicamente, no éramos de la misma opinión. Intentamos argumentar que por eso mismo era importante invertir tiempo y esfuerzo en tal reflexión, intentar una conversación a fondo que aportara respuestas, matices, una nueva comprensión, por ejemplo, del tópico mismo de la "colonización" soviética de la isla y muchos otros más.

Una de las consecuencias de la sospecha que el tema provocó a ambos lados del estrecho de la Florida fue la dificultad que tuvimos para conseguir fondos. No debe asombrarnos, entonces, que fuera una organización no cubana la que terminaría co-patrocinándolo, Trust for Mutual Understanding, una entidad privada ubicada en los EEUU dedicada al mejoramiento de las relaciones entre el antiguo campo socialista y los Estados Unidos. Otro detalle interesante es que el gobierno cubano tardara meses en otorgarles los así llamados "permisos de salida" a los invitados del simposio, un detalle este que también debe ser entendido en el marco de la política de George W. Bush, a la sazón presidente de los Estados Unidos, de no otorgarles visas a cubanos. El quid-pro-quo habitual entre los dos países estaba en juego, y según se nos dijo, el que un simposio de esa índole, que abordaría un tema todavía tan sensible fuera a celebrarse precisamente en los EEUU, era un elemento que agregaba dimensiones evocativas de este viejo conflicto.

Interminables deliberaciones fueron necesarias para terminar por moldear la idea del simposio: ¿cuál debía ser el número de ponentes?, ¿cuáles los campos a cubrir?, ¿por cuánto tiempo debería sesionar?, ¿cuál la extensión de las intervenciones? Lo otro importante fueron las sedes. La Universidad de Connecticut donde Loss es profesora no solo co-patrocinaría el simposio, albergaría la mayoría de las sesiones y el Instituto Cervantes de Nueva York patrocinaría una de las mesas. Finalmente, al cabo de varias semanas, tuvimos una lista de los participantes de nuestro simposio. Consideramos conveniente incluir todos sus nombres en esta relación por la naturaleza pionera del evento y también porque aunque no todas las ponencias fueron incluidas, por lógicas razones de espacio, en nuestro libro, Caviar with Rum: Cuba-USSR and the post-Soviet Experience, sí contribuyeron inmensamente al desarrollo de las temas abordados en él. ${ }^{2}$

${ }^{2}$ Véase el apéndice para la lista. 
El simposio superó nuestras expectativas; fue en realidad un momento de catarsis colectiva que nos señaló que habíamos dado comienzo a un examen más extenso y largo de todo el fenómeno de la amistad Cuba-URSS. Comenzaron a celebrarse en Cuba varios encuentros sobre el tema de la cultura soviética en la isla. A partir del 2007 en Cuba, se evidenció que enfrentarse en distintos foros con el legado soviético tenía un peso simbólico y estratégico nuevo. Por poner un solo ejemplo, los hijos de las uniones mixtas que en aquel entonces se encontraban buscando estrategias para documentar su presencia en la isla elevaron aún más su perfil. También a partir de 2007 diferentes ciclos sobre otros temas como la literatura rusa y exhibiciones que tenían como tema central la URSS y su impacto en Cuba tuvieron lugar, frecuentemente rescatando fuentes típicas del periodo soviético cubano y también letras cirílicas para anunciar sus eventos. Una solución con la que también nosotros habíamos dado para el diseño del póster con que anunciamos nuestro simposio, diseñado por Edvin Yegir, director del Design Studio en la Universidad de Connecticut, a quien le dimos varios ejemplares de la revista Sputnik para que se inspirara en ellos. En el 2009 la revista Temas llegó a organizar uno de sus debates sobre las huellas rusas y de Europa del Este en Cuba. Para coincidir con la Feria del Libro en 2010 cuyo país invitado fue Rusia, muchas revistas cubanas empezaron a dedicar números especiales a la Unión Soviética y Rusia; se publicaron libros con títulos difíciles de soñar como La cultura rusa en José Martí, se volvió a ver cine soviético y se empezó a ver por primera vez el cine ruso post-soviético en la televisión cubana y en jornadas en la pantalla grande. Al mismo tiempo, un fenómeno recíproco comenzó a darse dentro del ámbito ruso; la celebración de la presencia de la cultura cubana en aquél país, así como análisis y reflexiones públicas que buscaban enfatizar el impacto de la cultura rusa en Cuba.

\section{Caviar with Rum: Cuba-USSR and the post-Soviet Experience}

Desde el comienzo mismo imaginamos posible publicar un libro que compilaría algunas de las ponencias del simposio y la conferencia magistral. ${ }^{3}$ Elegimos un cuadro de otra de las panelistas, la pintora cubana Gertrudis Rivalta, para la portada. La imagen de la muchacha de ascendencia afrocubana con un elevado peinado en el que han instalado como adorno una reproducción en miniatura de una de las torres del Kremlin (de ahí el título del cuadro, "Quinceañera con Kremlin") condensa de manera magistral el tema del libro.

\footnotetext{
${ }^{3}$ José Quiroga, destacado especialista en cultura cubana, nos ofreció su ayuda en este proceso. Los profesores José Quiroga y Licia Fiol-Matta dirigen la serie New Directions in Latino American Cultures (Palgrave) en la cual fue publicado nuestro volumen.
} 
Pero otra vez, subestimamos el esfuerzo que significaría editar y publicar un libro de estas características. Tras dos años de interminables correos, del más delicado trabajo de edición, de la más exhaustiva y meticulosa revisión de las traducciones, ${ }^{4}$ de sopesar el balance de los textos, tras las comprensibles y mutuamente beneficiosas arduas negociaciones con la editorial, teníamos el libro. Sólo nos faltaba el título. Y tras otras muchas cavilaciones dimos con uno, Caviar with Rum. ¿Por qué ese título? Lo cierto es que queríamos un título, insistimos en ello antes los editores, que apelara a una audiencia mayor, que evitara la inevitable rigidez de un título más explicativo, que consideramos más adecuado usar como subtítulo. El título, tan sugerente, que aduce a la explosiva alianza que fraguaron, como dos avezados chefs de cocina, el joven Fidel Castro y el más experimentado Nikita Jruschev, es sin duda, una de las razones del atractivo del libro.

El libro contiene cinco secciones. La primera busca enmarcar la "ostalgie al estilo cubano" vista a través de ensayos de la bloguera (y arquitecta por profesión) Aurora Jácome, de la multipremiada poeta cubana Reina María Rodríguez y de Pedro González Reinoso, autor y artista de performance. Jácome, la creadora del primer y más citado blog sobre los "muñequitos rusos", los animados soviéticos que la televisión cubana programaba cotidianamente en los setentas y en los ochentas, explica por qué se sentía tan atraída por este universo y cómo precisamente se sentía más "en casa” viendo y recordando estos “muñequitos” y con sus coterráneos y contemporáneos que también los veían, que con el mundo español del fin de siglo veinte/ comienzo del veinte, donde le había tocado vivir por razones de su exilio a España. Por su parte Reina María Rodríguez evocaba la melancolía que le provocaba aquel mundo perdido, con el que dialogaba a través de imágenes concretas de la literatura contemporánea rusa y cubana. De Pedro González Reinoso, publicamos una versión abreviada de su libreto humorístico, Vidas de Roxy, que recrea la vida de una "rusa" abandonada por la URSS, curioso tópico que se repite en varios cuentos y documentales cubanos.

La siguiente sección, "Vasos comunicantes", abre con un texto de Juan Carlos Betancourt, un curador cubano que reside en Berlin, en el que señala de qué modo la Perestroika penetró e influyó las artes visuales cubanas. El crítico de arte, Ernesto Menéndez-Conde hace una lectura estratégica de un libro de Gerardo Mosquera de 1989 titulado El diseño se definió en octubre arguyendo que las ideas de este libro son cruciales para negociar los temas más polémicos de la cultura cubana actual, en particular cómo fue impactada negativamente por la sovietización. El escritor, ensayista y también traductor del ruso Jorge Ferrer, da un recorrido por el mundo soviético con una visión anti-nostálgica que recupera voces que discrepan de la nostalgia restorativa, por ejemplo, la del cineasta Roberto Fandiño y la del teórico y traductor Desidero Navarro que ha traducido, entre otros miles de textos, la escritura más

\footnotetext{
${ }^{4}$ Contamos con un excelente equipo de traductores.
} 
importante soviética formalista, de poco "uso" para estas estrategias "restaurativas". Del ensayista y profesor Carlos Espinosa, publicamos el ensayo "El mamut que se negó a extinguirse" en el que explica el proceso de rescate de Soy Cuba, sin duda la más importante co-producción cubano soviética. La tesis del ensayo de José Manuel Prieto es que el célebre poeta Heberto Padilla, quien fue la figura principal del por todos conocido "caso Padilla”, una redición de métodos estalinistas en la cultura cubana, modeló su posición crítica a la Revolución Cubana sobre la de los disidentes soviéticos que habían sido sus amigos en la lejana Moscú.

En la tercera sección, el "Ajiaco recalcitrante", Dmitri Prieto Samsonov y Polina Martínez Shvietsova, escritores de familias mixtas, cubanas y rusas, demuestran como lo "ruso" es sistemáticamente excluido del guisado nacional que simboliza la "cubanía". El ensayo visual del artista Tonel es una representación de encuentros imposibles y anacrónicos de figuras cubanas y soviéticas que llega a la médula de lo que llamamos una "transculturación recalcitrante". La pintora Gertrudis Rivalta Oliva, autora, como ya hemos dicho, del trabajo que figura en la portada de Caviar with Rum, explora en un texto muy personal, qué significaba para una cubana de la raza negra asimilarse a los paradigmas de la belleza soviética que las revistas de aquel país, de amplia circulación en la isla, proponían quizá como un modelo a seguir. Jacqueline Loss se enfoca en la presencia de la figura de la matrioska en la producción cultural de varias mujeres cubanas.

En la cuarta sección "El tractor imaginario", la antropóloga cultural Ariana Hernández-Reguant examina cómo un sistema de incentivos morales, importado del modelo soviético, intentó paliar la escasez material en la isla. En su ensayo pionero publicado originalmente en la revista Temas en el 2004, reconocido escritor cubano de ciencia ficción, Yoss enumera un inventario imaginario de lo que "dejaron los rusos en Cuba".

En la última sección "El coqueteo diplomático y económico", el ex diplomático soviético en América Latina y el Caribe, Yuri Pavlov, explora la persistencia del modelo socialista importado de los soviéticos en Cuba, a pesar de la Perestroika y el así llamado proceso de "rectificación" cubano de mediado de los ochenta. Y por fin, Mervyn J. Bain experto en la política exterior cubana en la Universidad de Aberdeen, problematiza la idea de que los rusos abandonaron la isla económicamente a través de elucidar las diferentes fases de la colaboración económica después de la desintegración de la URSS.

Caviar with Rum quería ser un volumen inclusivo, pero a la vez, esta inclusividad no nos permitió profundizar lo suficiente en algunos temas. Por ejemplo, bien se podría haber dedicado otro ensayo al profundo impacto del cine soviético en Cuba aparte del iluminador texto de Carlos Espinosa sobre la producción rusa-cubana Soy Cuba de Mikhail Kalatózov. Del mismo modo y a sabiendas dejamos fuera 
varios temas como el vínculo entre los países del ex campo socialista y el ballet cubano, la profunda y fructífera relación que se dio en la esfera de deportes como el boxeo, el impacto de la enseñanza de tantos pedagogos soviéticos en los conservatorios cubanos, quienes aportaron su visión y un rigor particular en el campo de la enseñanza y la interpretación de la música clásica, ni tampoco abordamos las profundas presencias de lo soviético en el ejército cubano.

La cronología con que abre el libro fue una sugerencia de la editorial pero fue imposible no dejar fuera mucho de los hitos importantes de la relación, por el número infinito de hitos que podríamos incluir en ella. Terminó siendo muy selectiva y hasta pudiera decirse, altamente subjetiva, toda vez que fácilmente podría haber incluido otros muchos eventos dejados fuera como por ejemplo la construcción de la planta nuclear Juraguá y otros muchos momentos. De hecho, tenemos pensado crear una cronología digital más extensa y interactiva sobre el tema que tome en cuenta estas y otras hitos.

Otra de los temas debatidos y más bien ventilados de manera más informal entre nosotros fue hasta qué punto "el pueblo" había sido impactado por lo soviético. Algunos repetían el tópico del escaso contacto que el pueblo cubano había tenido con los ciudadanos soviéticos, suficiente, según ellos, para no dedicarle ninguna investigación al tema. Este prejuicio nos sigue, es una constante: siempre se nos pregunta -no importa que sean personas cuyo móvil no sea “ideológico"-, por el "pueblo", si al nivel del ciudadano de pie, se sintió esa influencia. Por eso pensamos que tiene sentido explorarlo brevemente aquí en este contexto ${ }^{6}$.

Lo cierto es que, sin lugar a dudas, la mayor influencia se dio entre las elites (la amistad del poeta cubano Heberto Padilla con el célebre bardo ruso Evgueny Evtushenko es un claro ejemplo de ello), esa transmisión de la cultura de la disidencia que documenta José Manuel Prieto en su ensayo "Padilla, el primer disidente" que puede decirse que hoy día continúa de algún modo si uno analiza la relación existente y el apoyo de tantos países del Este actual en la oposición cubana. El poeta y también escritor de novelas policíacas, Luís Rogelio Nogueras luego fue un gran lector y rendido admirador del autor

\footnotetext{
${ }^{5}$ Habiendo aparecido primeramente en Todos se van (2007) de Wendy Guerra, una novela que Loss, de hecho, analiza en su ensayo en Caviar with Rum, la central nuclear es el escenario de la reciente película de Carlos Machado Quintela La obra del siglo (2015) y también del documental del Adrian Silvestre y Luis Alejandro Yero, Natalia Nikolaevna (2014), datos que señalan la importancia de este sitio en el imaginario cubano.

${ }^{6}$ No llegamos a problematizar en Caviar with Rum esta dicotomía popular/elitista y la influencia soviética. El caso de ciencia ficción, por ejemplo, ha sido ampliamente estudiada por Yoss y Juan Carlos Toledano, pero es solo uno de los muchos géneros en los que se observa este claro fenómeno de "ósmosis". Sin embargo, los ejemplos que también ilustran este fenómeno son abundantes pero a la fecha siguen sin haber sido estudiados adecuadamente.
} 
soviético Vladímir Bogomolov, autor de, entre otras, En agosto de 1944. ${ }^{7}$ Uno puede rastrear esa influencia y otras influencias (pienso también los libros del novelista búlgaro y teórico de la novela negra Bogomil Rainov, ampliamente leído en Cuba) en las obras de Nogueras en particular en su célebre novela Y si muero mañana de 1978 que gozó de amplia aceptación a nivel popular en Cuba e ilustra de qué modo la influencia soviética y del Este iba más allá de las élites, se penetraba al nivel "popular" esta manera que pudiéramos llamar "osmótica".

Transferencias semejantes ocurrieron también en el campo de las artes audiovisuales. Nos referimos, por ejemplo, a la serie televisiva soviética, 17 instantes de una primavera, basada en la novela del escritor Yulian Semionov y qué gozó de alta aceptación popular en Cuba. Y bien, sin la influencia de esa serie televisa que cuenta las vicisitudes del agente ruso Stierlitz en el Berlín del Tercer Reich en lo más álgido de la Segunda Guerra Mundial, pensamos que no hubiera existido el todavía más popular serial cubano En silencio ha tenido que ser, que narra las vicisitudes de un agente de la inteligencia cubana infiltrado en los Estados Unidos. La influencia del serial soviético en el cubano es visible no solo en cuanto a la narrativa sino también en detalles menores como la música de los hermanos José María y Sergio Vitier, que guarda similitud en cuanto a la intención de grandeza y elegancia casi clásica con la de la música jazzeada del compositor soviético Mikael Tariverdiev, autor del tema musical de 17 instantes de una primavera.

La misma transferencia es observable en películas cubanas como La muerte de un burócrata, cuyo tema parece salido directamente del arsenal ideológico soviético: las intenciones del partido son siempre buenas, son los malvados burócratas quienes las tergiversan, son ellos los verdaderos culpables, de que las cosas no funcionen bien en el paraíso socialista. Otra influencia de la misma índole es observable en un filme que terminó siendo de amplio consumo popular. Nos referimos a Las doces sillas (Tomás Gutiérrez Alea, 1962), cuyo guión está sacado de la novela de los escritores soviéticos de los años veinte, Ilya Ilf y Evgeny Petrof. Ahora bien, las andanzas de Ostap Bender, Oscar en la versión cubana, actuado por Reynaldo Miravalles, son muy recordadas por los cinéfilos cubanos. No importa que para muchos de ellos el origen del libreto, su profunda inspiración soviética, no sea ya hoy tan evidente.

\footnotetext{
${ }^{7}$ De hecho, el 7 de diciembre del 1984, Bohemia publicó una entrevista que Nogueras le hizo a Bogomolov en la cual el autor cubano reconoce que su primera pregunta sobre el género policial incomoda a Bogomolov debido a los "prejuicios elitistas" hacia el género. La recién fallecida Zoia Barash, ucrania que residió en Cuba durante aproximadamente cuarenta años, tradujo En agosto de 1944 y otros libros importantes de la literatura soviética.
} 
Jacqueline Loss y José Manuel Prieto. Caviar con ron...

\section{Otra vez la nostalgia}

La recepción de Caviar with Rum fue entusiasta. Los reseñadores elogiaron la heterogeneidad de los ensayos (Román de la Campa) y el hecho de que es un libro que ha abierto nuevas líneas de pensamiento (Roberto Madrigal y Oxana Álvarez). Es más hoy en día hay muchos estudiosos que están desarrollando proyectos muy interesantes sobre la relación entre Cuba y la Unión Soviética en la cultura, varios de ellos incluidos en esta revista, pero cada día tomamos nota de nuevas investigaciones en un creciente número de campos que buscan profundizar los vínculos históricos entre Cuba y el campo socialista. Es decir, más que nunca, el enfoque de análisis de Caviar with Rum sigue vigente frente a una geopolítica con la cual nunca podíamos soñar en 2004.

Como ya dijimos al comienzo, Putin viajó a Cuba con la buena nueva de que condonaría la vieja multimillonaria deuda de los cubanos con el imperio soviético. Este condono, el compromiso de reactivar la exploración petrolera y de reinvertir unos 3.500 millones de la deuda en proyectos de desarrollo en la isla fueron sólo los últimos en una serie de gestos de "fraternidad reavivada" que incluyen la consagración de la iglesia rusa ortodoxa en la Habana en 2008, el pacto firmado entre Raúl Castro y Dimitri Medvedev en 2009 para reforzar las relaciones bilaterales y un incremento de inversiones en planes educativos a partir del 2010 -el mismo año que Cuba invitó Rusia como país de honor a la Feria del Libro.

La feria constituyó un espectáculo curioso. El visitante podía encontrar a rusos de mediana edad, bailando una salsa que no sólo les recordaba sus viajes a la isla de veinte años atrás, sino que también, era visible, embargados por la sensación de haber regresado a un espacio familiar, que les recordaba sus propias agridulces experiencias bajo el comunismo.

Vimos también a cubanos de su edad acercárseles para hablar un idioma que ciertamente había perdido relevancia a partir de 1992, fecha en que las palabras "la amistad fraternal y la cooperación de la Unión Soviética” fueron borradas de la Constitución cubana. Quien hubiera podido imaginar que la frase: "Todas queremos ser como Valentina Tereshkova" que figura en un díptico del 2004 de Gertrudis Rivalta, sería resucitada algún día. De hecho, fue la misma Tereshkova, la más que célebre mujer cosmonauta, quien en su calidad de legisladora, durante una sesión de la Duma a principios de julio de ese mismo 2010 recordó a los rusos la hospitalidad de los cubanos tras el desastre de Chernobyl en 1986, cuando la isla caribeña aceptó a miles de niños soviéticos (según algunos cálculos entre 24.000 y 40.000), que fueron recibidos en Tarará, al este de la ciudad de la Habana, donde recibieron atención médica calificada y pudieron descansar de los traumas de la horrible tragedia nuclear. Mientras algunos de los términos clave del viaje de Putin sonaban casi anticuados -el progreso en los campos de energía, 
transporte y el espacio exterior-, la persistencia de los rusos en Cuba en tiempos actuales después de la supuesta distensión entre Cuba y Estados Unidos y Rusia es innegable. La llegada del buque espía ruso a la Habana en enero del 2015 o la visita unas semanas después del ministro de Defensa de Rusia, Serguéi Shoigu, a la isla donde afirmó: "Nuestros países están ligados por un interés común de crear un mundo justo y policéntrico con un orden basado en la igualdad, el respeto al derecho internacional y el papel central de Naciones Unidas" son sólo algunos de los muchos ejemplos de una Rusia no tan dispuesta a despedirse de su viejo aliado geopolítico mucho menos en un contexto en que algunos de los países vecinos de la ex URSS están enfrascados en álgidos conflictos militares. Lógicamente, a pesar de los acercamientos de los últimos años, de la tan publicitada visita de Putin, la relación de Rusia con Cuba jamás será la de antes: falta la convicción, el calor ideológico si se quiere, el claro elemento mesiánico de los rusos y el franco respeto de los cubanos, que durante años fueron promovidos por ciertos entusiastas como una suerte de hermanos mayores, dignos de imitación. Rusia, por otra parte, no es la URSS, sus recursos son más limitados y en nada están dispuestos a relaciones basadas en un componente primordialmente ideológico. Lo que prima ahora es cierta nostalgia imperialista de parte de Putin, que sigue empecinado en enmendar lo que Gorbachov deshizo, la grandeza soviética.

El tema, algo de espera luego de casi cincuenta años de las más estrechas relaciones, de las vidas entrelazadas entre ambos países, es virtualmente inagotable. Las respuestas que ha suscitado nuestro libro, tanto a nivel académico, como esa revaloración más reciente de todo lo soviético, nos lleva a volver a cambiar la pregunta de Yoss de ¿qué dejaron los rusos? a “ ¿acaso se fueron realmente?”. 


\section{Bibliografía}

Álvarez, Oxana. "Review of Caviar with Rum". Transmodernity: Journal of Peripheral Cultural Production of the Luso-Hispanic World 3, 2 (2014).

Boym, Svetlana (2001). The Future of Nostalgia. New York: Basic Books.

De la Campa, Román. "Review of Caviar with Rum: Cuba-USSR and the post-Soviet Experience" Review: Literature and Arts of the Americas 47, 1 (2014): 119-120.

Loss, Jacqueline y Prieto, José Manuel (eds.) (2012). Caviar with Rum: Cuba-USSR and the post-Soviet Experience. New York: Palgrave.

Madrigal, Roberto. "Lo que el sóviet nos dejó” Diletante sin causa (2012).

\section{Noticias en medios y sitio web}

"Estados Unidos es incapaz de enemistar a Rusia y Cuba" Sputnik News (2015).

"Rusia y Cuba desarrollan una 'constructiva' cooperación militar” HispanTV(2015).

Nazdarovie

KAMCHATKA 5 JULIO 2015 


\section{Apéndice}

1. Raúl Aguiar, escritor cubano y también especie de rusófilo desde una edad temprana. Ha escrito sobre los lazos en la ciencia ficción entre Cuba y la Unión Soviética. Por no haber recibido la visa no pudo participar en el congreso.

2. Carlos A. Aguilera escritor cubano que actualmente vive en la república checa, cuyas publicaciones incluyen El imperio Oblómov, una novela que curiosamente empieza así: "Ahora hablemos de mi odio hacia el Este".

3. Isabel Álvarez Borland, especialista en literatura cubana americana y latina en College of the Holy Cross, autora de Negotiating Identities in Cuban American Art and Literature and Cuban-American Narratives of Exile: From Person to Persona, entre muchas otras publicaciones.

4. Nadya Bakuradze, gestora cultural en la capital rusa que organizó el primer evento cultural ruso no estatal en Cuba en 2005

5. Mervyn J. Bain, académico en la Universidad de Aberdeen que publicó Soviet-Cuban Relations 1985 to 1991. Changing Perceptions in Moscow (2007) y Havana, and Russian-Cuban Relations Since 1992. Continuing Camaraderie in a Post-Soviet World en 2008.

6. Juan Carlos Betancourt, curador cubano de arte, residente en Berlin, que también ha publicado un artículo interesante sobre la obra de José Manuel Prieto y de Iván de la Nuez, titulado "La Missachtung de la nostalgia como estrategia creativa en dos obras literarias de la diáspora cubana de los 90 ”.

7. Antonio Eligio Fernández (Tonel), reconocido pintor cubano, residente en Vancouver, cuya obra suele chotear con los elementos más irónicos de la extraña “fraternidad”.

8. Jorge Ferrer, escritor cubano, autor de Minimal Bildung (2001) entre varios otros textos y traductor apremiado del ruso, residente en Barcelona..

9. Anke Finger, profesora en la Universidad de Connecticut, especialista en la obra de Vilém Flusser, que analizó en ese foro la creación de la memoria y Alemania del Este desde una perspectiva personal. 
10. Víctor Fowler Calzada, ensayista, crítico y escritor apremiado para numerosos libros incluyendo los poemarios, La maquinista de Auschwitz (2004) y La obligación de expresar (2008), que iba a dar una conferencia en la mesa titulada "Cartografías de ciudadanía".

11. Omar Godinez, pintor cubano, residente en Rusia, que ejerce un rol importante en la diseminación de la cultura cubana en Rusia.

12. Pedro González Reinoso, artista performático residente en Cuba--aunque los EEUU no lo permitieron llegar para el congreso, sí, llegó a dar su performance extraordinario de "La Rusa Roxana Rojo” en la Universidad de Connecticut tres años después en 2010.

13. Ariana Hernandez-Reguant, antropóloga cultural que compiló y editó Cuba in the Special Period (2008) entre otros textos importantes sobre este período.

14. Aurora Jácome, arquitecta cubana residente en España que lanzó la bitácora más importante sobre la nostalgia cubana hacia los muñequitos rusos-http:// munequitosrusos.blogspot.com/.

15. Suki John, coreógrafa, académica en Texas Christian University y autora de Contemporary Dance in Cuba.

16. Christopher Larkosh, profesor en University de Massachusetts-Dartmouth, que ha publicado numerosos textos sobre las teorías de la traducción y presentó "Magyar film (A Rereading of Soviet Space)” en el simposio.

17. Polina Martínez Shvietsova, narradora, poeta, artista performática y periodista cubana rusa, cuyo ensayo sobre la diáspora post-soviética en Cuba escrito con Dmitri Prieto Samsonov fue leído in absentia.

18. Ernesto Menéndez Conde, académico y escritor cubano residente en New York, especialista en artes visuales y director de art experience:nyc.

19. Jorge Miralles, traductor y autor cubano de Las voces del pantano, que buscó trazar en su presentación (leída in absentia) un inventario personal de lo soviético.

20. Desiderio Navarro, el fundador de la revista Criterios que durante cuatro décadas ha traducido y publicado la teoría más interesante del mundo eslavo, la serie de libros Criterio 
Jacqueline Loss y José Manuel Prieto. Caviar con ron...

(1994) y del e-zine denken pensée thought mysl en 2011. Por no haber recibido la visa no pudo participar en el congreso.

21. Veronica Pérez Konina (Proskurnina), escritora cubana rusa residente en Moscú cuya visión personal sobre su identidad híbrida puede encontrarse en un ensayo interesante suyo publicado en The Portable Island: Cubans at Home in the World.

22. Nikola Petkovic, académico y escritor croata de numerosos ensayos, novelas y poemarios, que nos habló de la relación entre Tito y Castro.

23. Dmitri Prieto Samsonov, pensador cubano ruso cuya presentación con Polina Martínez Shvietsova sobre la diáspora post-soviética en Cuba fue leída in absentia.

24. José Quiroga, autor de los muy conocidos y citados estudios Cuban Palimpsests (2005) y Tropics of Desire: Interventions from Queer Latino America (2001) entre muchos otros clásicos, dio la conferencia magistral.

25. Gertrudis Rivalta, artista visual que nos prestó generosamente su cuadro espectacular "Quinceañera con Kremlin” para la portada de Caviarwith Rum.

26. Ernesto René Rodríguez, cinematógrafo y co-director de 9550 , el primer documental que exploró la herencia soviética en Cuba. Se estrenó en el marco del simposio aunque su director no estuvo presente.

27. Reina María Rodríguez, poeta internacionalmente apremiada cuya presentación sobre la nostalgia, fue leída in absentia.

28. Rafael Rojas, uno de los académicos más famosos de su generación, prolífico, entre los más recientes, La vanguardia peregrina.: El escritor cubano, la tradición y el exilio (2013). De su pluma también, un ensayo interesante que se titula "Souvenirs de un Caribe soviético" (2008), el cual busca elucidar las lecturas soviéticas entre los cubanos.

29. José Miguel Sánchez (Yoss), escritor cubano de ciencia ficción cuyo artículo "Lo que dejaron los rusos" publicado en 2004 en Temas fue uno de los primeros en aparecer sobre el tema. Su conferencia fue leída in absentia.

30. Rolando Sánchez Mejías, fundador en 1993 en la Habana del grupo Diásporas cuyas publicaciones incluyen Historias de Olmo (2001), Cuaderno de Feldafing (2003) que habló sobre los mecanismos de poder heredados de los soviéticos. 
31. Vladimir Alexander Smith Mesa, cubano residente en Londres que presentó una conferencia original sobre los carteles para el cine del viejo campo socialista en Cuba; en 2011 publicó su tesis doctoral titulada KinoCuban: the Significance of Soviet and East European cinemas for the Cuban Moving Image.

32. Juan Carlos Toledano, profesor en Lewis \& Clark College que es especialista en ciencia ficción cubana y autor de "From Socialist Realism to Anarchist-Capitalism: Cuban Cyberpunk" (2005) entre otros textos.

33. Anna Lidia Vega Serova. escritora cubana nacida en Leningrado que publicó entre muchos otros libros su novela Ánima fatua, un especie de bildungsroman que se enfoca en la experiencia de un sujeto híbrido entre Cuba y la Unión Soviética. Vega Serova. Aunque no le dieron la visa en 2007, desde aquel entonces, ha llegado a visitar la Universidad de Connecticut.

También contamos con el apoyo de varios profesores latinoamericanistas y caribeñistas de la misma Universidad de Connecticut y en la zona, que sirvieron de moderadores y tomaron parte muy activa en el simposio. Nos referimos a Esther Allen, Odette Casamayor-Cisneros, Robin Greeley, Guillermo Irizarry, Melina Pappademos y Esther Whitfield. 